\title{
A High-Echoic Layer Surrounding the Heart Suggesting Cardiac Tamponade by Clotting
}

\author{
Ikuto Takeuchi, MD ${ }^{1}$ Jun Shitara, MD, $\mathrm{PhD}^{1} \quad$ Youichi Yanagawa, MD, $\mathrm{PhD}^{1}$ (1) \\ 1 Department of Acute Critical Care Medicine, Juntendo Shizuoka \\ Hospital, Izunokuni City, Shizuoka Prefecture, Japan \\ Address for correspondence Youichi Yanagawa, MD, PhD, 1129 \\ Nagaoka, Izunokuni City, Shizuoka 410-2295, Japan \\ (e-mail: yyanaga@juntendo.ac.jp).
}

Aorta (Stamford) 2021;9:116-117.

\author{
Abstract \\ Keywords \\ - aortic dissection \\ - tamponade \\ - Loeys-Dietz \\ syndrome
}

\begin{abstract}
A 16-year-old boy experienced a sudden loss of consciousness. On arrival, he was in cardiac arrest. An ultrasound study revealed a high-echoic layer surrounding the heart. He received a diagnosis of clotting cardiac tamponade. Urgent thoracotomy with pericardiotomy was performed, but he failed to obtain return of spontaneous circulation. Physicians should focus on not only low-echoic but also high-echoic areas to accurately diagnose clotting, which can result in a critical condition if not managed properly.
\end{abstract}

A 16-year-old boy experienced a sudden loss of consciousness at home in front of his parents. On arrival, he was in cardiac arrest with pulseless electrical activity. An ultrasound study revealed a high-echoic layer surrounding the heart with a high-echoic layer surrounding the ascending aorta as well (-Figs. 1 and 2). He received a diagnosis of

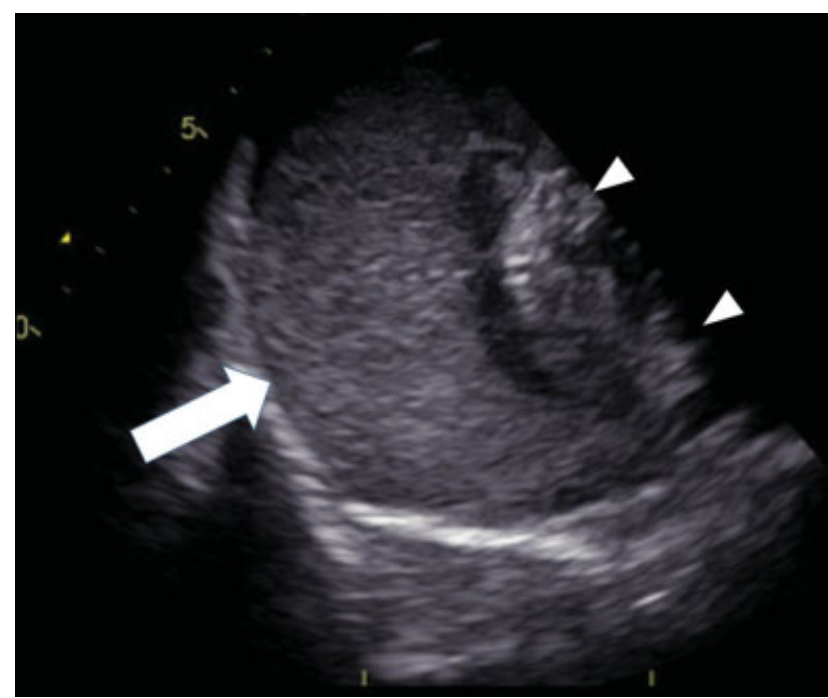

Fig. 1 The ultrasound study revealed a high-echoic layer (arrow) surrounding the heart (arrowheads) suggesting clotting cardiac tamponade.

received

April 2, 2020

accepted after revision

February 25, 2021

published online

October 12, 2021 clotting cardiac tamponade. Urgent thoracotomy with pericardiotomy was performed ( $\mathbf{- F i g . 3}$ ), but he failed to obtain return of spontaneous circulation. Autopsy imaging indicat-

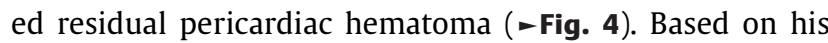
features, he was suspected of having Loeys-Dietz or Marfan syndrome. We postulated that connective tissue disease had induced Type A aortic dissection with subsequent rupture that had resulted in cardiac tamponade and cardiac arrest.

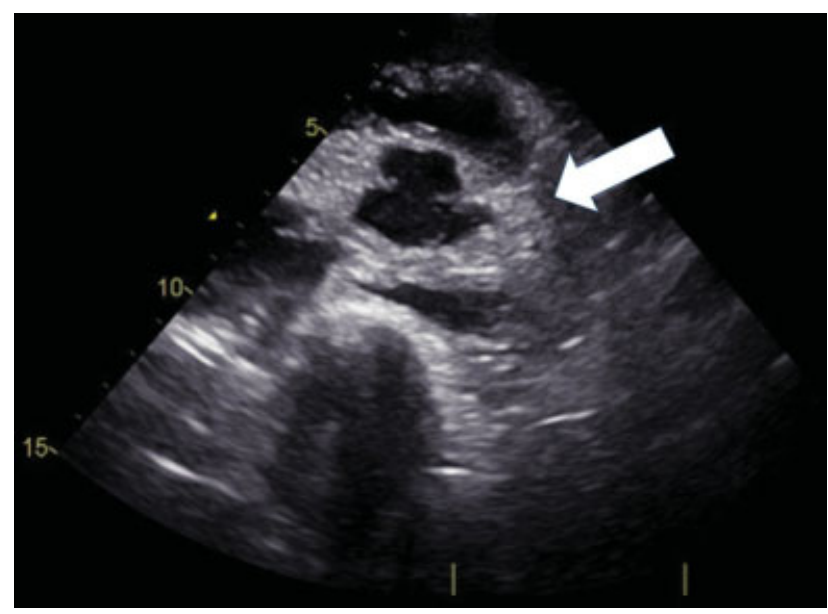

Fig. 2 The ultrasound study revealed a high-echoic layer (arrow) surrounding the ascending aorta, suggesting aortic dissection.

\section{(C) 2021. The Author(s).}

This is an open access article published by Thieme under the terms of the Creative Commons Attribution License, permitting unrestricted use, distribution, and reproduction so long as the original work is properly cited. (https://creativecommons.org/licenses/by/4.0/)

Thieme Medical Publishers, Inc., 333 Seventh Avenue, 18th Floor, New York, NY 10001, USA 


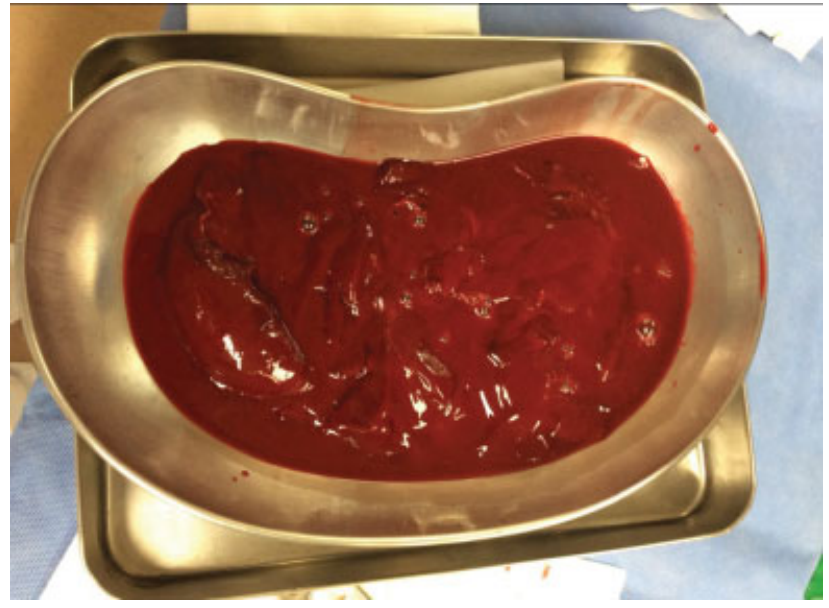

Fig. 3 Urgent thoracotomy with pericardiotomy revealed a clot weighing approximately $1 \mathrm{~kg}$.

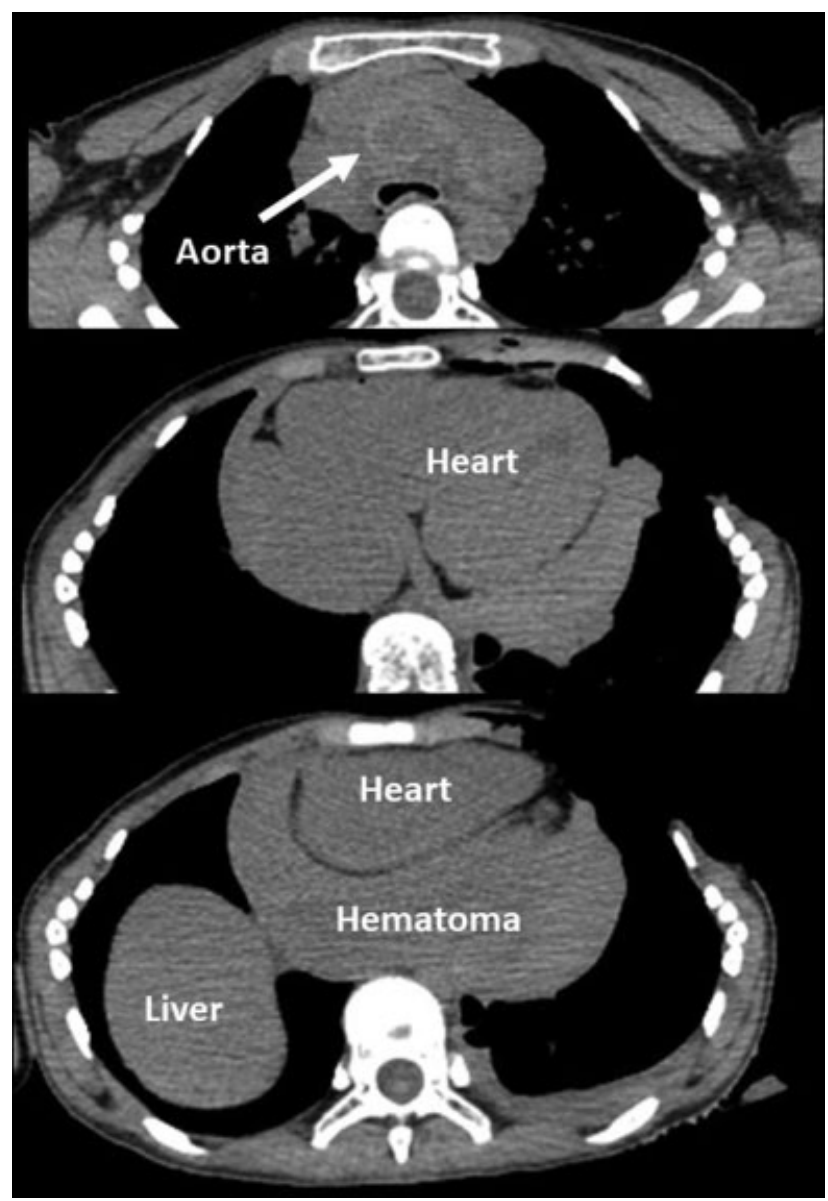

Fig. 4 Autopsy imaging. Computed tomography indicated residual pericardiac hematoma.
Permission to perform a genetic analysis was not obtained from his parents.

In cases of hemorrhaging into a closed space, the blood forms a blood clot to achieve hemostasis. During this process, the whole blood separates into a blood clot and serum. When the serum accumulates in one space, it may be detected as fluid by ultrasound. However, it takes some time for the serum to accumulate. Accordingly, a focused assessment with sonography in trauma, which focuses on low-echoic areas to detect serum, is well known to have a high falsenegative rate for the acute hemorrhaging state in traumatized patients. ${ }^{1}$ Clots are scanned as high-echoic areas on ultrasound studies. If physicians focus not only on lowechoic but also high-echoic areas, the sensitivity for detecting clinically significant blood clots in the human body may be improved.

Unfortunately, the present patient failed to obtain a favorable outcome; however, the urgent resolution of cardiac tamponade by clotting and subsequent treatments might result in a favorable outcome in other patients. ${ }^{2,3}$

\section{Funding}

This work was supported in part by a Grant-in-Aid for Special Research in Subsidies for ordinary expenses of private schools from The Promotion and Mutual Aid Corporation for Private Schools of Japan (http://dx.doi. org/10.13039/501100012359).

\section{Conflict of Interest}

The authors declare no conflict of interest related to this article.

\section{Acknowledgment}

None.

\section{References}

1 Do WS, Chang R, Fox EE, Wade CE, Holcomb JB, Martin MJNCTH Study Group. Too fast, or not fast enough? The FAST exam in patients with non-compressible torso hemorrhage. Am J Surg 2019;217(05):882-886

2 Keiko T, Yanagawa Y, Isoda S. A successful treatment of cardiac tamponade due to an aortic dissection using open-chest massage. Am J Emerg Med 2012;30(04):634.e1-634.e2

3 Yatsu S, Yanagawa Y, Nagasawa H, Tambara K, Suwa S. Successful treatment of cardiac tamponade due to rupture of the heart using open-chest pericardiotomy. Cureus 2020;12(02):e7101 